\title{
NUOVE PROSPETTIVE NEGLI STUDI ECONOMICI SULL'ILLUMINISMO LOMBARDO
}

\author{
PIER LUIGI PORTA (*)
}

\begin{abstract}
RiAssunTO. - Gli studi sull'Illuminismo (e sull'Illuminismo italiano in particolare) hanno conosciuto in Italia una stagione assai fertile in questo dopoguerra con una forte motivazione a ricercare modelli di convivenza civile, di cultura e di valori fondanti per le nostre società uscite dai grandi traumi collettivi del secolo passato. L'economia politica - senza dubbio tra le presenze scientifiche di maggiore spicco nei diversi Illuminismi sulla scena europea - è molto spesso rimasta in secondo piano. Il Convegno promosso dall'Istituto Lombardo nel dicembre 2011 ha inteso raccogliere una serie molto ampia di spunti e di indagini, espressione della maturazione a livello internazionale di nuove prospettive di studi sull'Illuminismo concepiti a partire dalla economia politica. L'Illuminismo lombardo, all'interno del contesto italiano, rappresenta un caso emblematico per questo genere di studi.

In questa prospettiva il presente contributo si informa prevalentemente al canone storico-analitico schumpeteriano. Questo implica anche che l'argomentazione coinvolga differenti periodi storici ed è intesa ad individuare uno o più fili conduttori capaci di riassumere i caratteri identitari della tradizione italiana di economia politica in un senso generale. In questo quadro l'Illuminismo lombardo acquista un importante valore idealtipico.
\end{abstract}

$$
* * *
$$

ABSTRACT. - New perspectives in the economic studies of the Lombard Enlightenment.

Since World War II there has been an increasing interest in the Italian Enlightenment. This could be partly explained as a response to the emerging need for deeper understanding of the roots of Western culture and of the civic values that had been so much shattered by the traumatic experiences of a new kind of war ravaging Europe and other parts of the world. Political Economy - that was originally called Civil Economy in eighteenth century Naples - was indeed prominent in the Italian Enlightenment, although that historical fact has only been imperfectly reflected in

(*) Istituto Lombardo Accademia di Scienze e Lettere; Università degli Studi, Milano-Bicocca. 
much of recent historiography. There is, however, a new developing line of investigation that puts Political Economy at the core of research into the Italian enlightenment.

The Conference sponsored by the Istituto Lombardo Accademia di Scienze e Lettere focussed precisely on the Political Economy that featured so prominent in the theoretical contributions and policy proposals of the Lombard Enlightenment.

This essay mainly reflects the canon of the Schumpeterian approach to the history of economic analysis. This implies the consideration of different historical periods with the aim of bringing out those research strands that contribute to the identity of the Italian tradition of economic thought and analysis.

\section{INTRODUZIONE}

Gli studi sull'Illuminismo hanno conosciuto in Italia una stagione assai fertile in questo dopoguerra con una forte motivazione a ricercare modelli di società, di cultura e di valori civici fondanti per le nostre società uscite dai grandi traumi collettivi del secolo passato. Nel dicembre 2011 è mancato Furio Diaz, certamente uno dei più attivi in questo senso tra i cultori di storia moderna.

L'economia politica - senza dubbio tra le presenze scientifiche di maggiore spicco nei diversi Illuminismi sulla scena europea - è sostanzialmente rimasta in secondo piano in molti degli studi sull'Illuminismo. In qualche misura in ombra sono restati non pochi contributi di taglio storico-analitico nel senso schumpeteriano del termine. Il disegno di questo Convegno ha inteso raccogliere una serie, in realtà molto ampia, di spunti e di indagini nella convinzione che al presente sia in fase di maturazione a livello internazionale una nuova prospettiva di studi sull'tlluminismo la quale muove i passi proprio dalla economia politica. L'Illuminismo lombardo, all'interno del contesto italiano, rappresenta un caso emblematico per questo genere di studi.

Sarà compito della silloge che uscirà dal Convegno tracciare un bilancio e valutare il tentativo che qui è stato fatto di sostanziare la accennata tesi generale circa la lezione dell'Illuminismo lombardo, una tesi emersa in questa sede col concorso di tutti i contributi presentati e messa in rilievo, in primo luogo, dalla relazione introduttiva presentata da Roberto Scazzieri.

In sede conclusiva può essere opportuna una sintetica analisi delle condizioni attuali e di alcuni mutamenti in corso negli studi storico-analitici in economia che possono contribuire a spiegare la mutata temperie culturale e gettare luce sulle potenzialità della storia della analisi econo- 
mica. In questa prospettiva il taglio di questo contributo sarà probabilmente più decisamente informato al canone storico-analitico di quanto non sia accaduto per i contributi che lo hanno preceduto nel corso del Convegno. Questo implica, tra altre cose, che l'argomentazione si sposterà su diversi periodi storici e cercherà di individuare uno o più fili conduttori capaci di riassumere i caratteri identitari della tradizione italiana di economia politica in senso generale. In questo quadro l'Illuminismo lombardo acquista, come accennato, un valore idealtipico.

$\mathrm{Si}$ è sostenuto di recente che la intera tradizione di pensiero economico italiano trova la sua forma caratteristica nel canone della economia civile, espressione di una triplice combinazione di elementi: una concezione dell'ordine politico-economico come realtà che scaturisce dalla società civile contrapposta alla autorità politica ossia allo Stato; una visione continuamente riformatrice; un impegno scientifico-culturale della disciplina rivolto prevalentemente ad una ricerca suscettibile di spendibilità applicativa. I temi salienti della ricchissima tradizione italiana negli studi economici toccano un insieme di problemi tra i quali i principali sono i seguenti: a) la preoccupazione etico-sociale, che pervade l'opera degli economisti italiani in ogni tempo e produce teoria orientata alla azione e impostata in modo tale da rendere 'naturale' la analisi delle istituzioni e in generale la relazione con gli aspetti extra-economici; b) un diretto e approfondito interesse per l'economia dinamica; c) una impostazione di metodo basata sulla stretta fusione tra la disciplina e la sua storia, così che l'interesse per la storia dell'analisi economica costituisce esso stesso un elemento distintivo della tradizione italiana. ${ }^{1}$

Il riferimento alla vita civile costituisce un elemento significativamente ricorrente nella tradizione italiana come componente della storia delle idee che interseca gli studi economici. Menzioniamo qui in breve gli esempi classici di autori ben noti anche distanti nel tempo come Matteo Palmieri, Poggio Bracciolini, Leonardo Bruni fino a Paolo Mattia Doria, come hanno mostrato classici studi da Jacob Burckhardt a Hans Baron a Eugenio Garin. L'epoca illuminista, soprattutto nella seconda parte del XVIII secolo, costituisce forse il momento più interessante, quando la categoria del 'civile' si associa direttamente coll' $e$ conomico dando vita a un paradigma di successo e di rilievo come filo conduttore di una serie di studi che attraversano la storia delle idee eco-

1 Dizionario della economia civile (2009); Faucci, 2000, Bellanca, 2000. 
nomiche in Italia. Da Muratori in poi il tema della pubblica felicità prende forza precisamente come fondamento della nuova economia politica dell'età delle riforme, producendo un canone destinato ad avere una sua continuità e una collocazione duratura specie (ma non soltanto) all'interno della tradizione italiana.

Per affrontare il problema con specifico riferimento al contesto dell'Illuminismo lombardo, occorre svolgere l'analisi alla luce dei due sviluppi seguenti: 1) ricordare e chiarire taluni aspetti della rilettura del pensiero economico classico, affermatasi in anni recenti 2) accennare, con qualche approfondimento, alla ricostruzione in corso della linea di sviluppo storico della tradizione italiana in economia politica. Il revisionismo storico ha raggiunto qui - nel corso degli ultimi trenta anni risultati notevoli.

Osserviamo anche, in premessa, che esistono diversi modi di intendere il termine civile o civico applicato alla tradizione del pensiero economico. Essi sono, schematicamente, i seguenti: 1) la concezione civica del repubblicanesimo machiavelliano 2) la idea classica del 'buon governo' riconducibile ultimamente alla oikonomia greca 3) la nozione della economia civile, come forma di ordine economico spontaneo, introdotta da Genovesi.

\section{L'ECONOMIA CIVILE: UN'INDAGINE SU CIÒ CHE TIENE ASSIEME IL CORPO SOCIALE}

Alla base della concezione della economia civile vi è una concezione dell'ordine politico-sociale che ha al suo centro la idea di vita activa collegata con il vivere civile. John Pocock ha teorizzato quella concezione come radice dell'ideale della virtù del cittadino repubblicano che ritrova la sua identità nella coscienza civica di appartenenza alla comunità attraverso l'esercizio del vivere civile. Il lavoro di Pocock venne descritto all'epoca come una magistrale rassegna dell'intera tradizione dell'umanesimo civico dalle sue origine fiorentine (Machiavelli) sino ai suoi sviluppi successivi nel pensiero politico anglo-americano prima e dopo la rivoluzione americana (Winch, 1978, p. 33). Pocock riprende anche analisi di Garin e altri. E' una concezione che conduce ultimamente a contrapporre ricchezza e virtù.

Da un'altra prospettiva la economia civile può essere intesa come l'esito di una forma di buon governo. La cosiddetta Hausväterliteratur, 
originata e sviluppata soprattutto in Germania, ha sviluppato il concetto estendendo alla collettività politica (la polis) la oikonomia aristotelica e generando così, appunto, l'economia politica.

In questa sede siamo piuttosto interessati a una terza modalità di intendere il concetto di economia civile: è la forma che emerge nella letteratura settecentesca e che contribuisce a consolidare il paradigma dell'ordine spontaneo della società commerciale, dove il commercio diventa quella specifica forma di mutuo soccorso che consente a una società complessa non solo di sopravvivere, ma di crescere, all'interno della continua interazione tra mercato e istituzioni.

$\mathrm{Vi}$ è storicamente un complesso itinerario che conduce dalla ben nota dicotomia di Hobbes (stato di natura/società civile) alla tricotomia che è alla radice della concezione dell'ordine politico-sociale caratteristica della economia politica della Scuola classica nella seconda metà del Settecento: si tratta di una concezione fondata sui tre elementi dello stato di natura, società civile, stato. La ricostruzione che mette al centro il concetto di economia civile approda a ritrovare le radici fondanti dell'economia di Smith proprio nella tradizione italiana, anziché negli Economisti francesi (i fisiocrati) come più spesso si ritiene malgrado le esplicite prese di distanza dell'autore. ${ }^{2}$ Essa approda altresì (da una diversa prospettiva) a rivelare una cesura importante, seppure a lungo sottovalutata, all'interno della Scuola Classica medesima.

Se infatti quest'ultima Scuola appare da un lato unificata da una comune concezione del valore, essa è al suo interno profondamente differenziata per altri non meno importanti aspetti. Vi è infatti, da una parte, l'ottimismo di Smith e dall'altra parte il pessimismo della 'scienza triste' di Ricardo. Si tratta di una divisione fondamentale, che oggi riemerge prepotentemente, per esempio, attraverso gli sviluppi della dinamica economica strutturale, ispirata espressamente al paradigma smithiano, dopo anni nei quali la rivisitazione della economia classica

2 "Sembra che Quesnay, - scrive ad es. Smith (IV.9) - il quale era medico e medico molto profondo, abbia...immaginato" che il corpo politico (al pari del corpo umano) "possa fiorire e prosperare soltanto con un certo preciso regime, quello della perfetta libertà e giustizia. Sembra che egli non abbia considerato che nel corpo politico lo sforzo naturale che ognuno fa continuamente per migliorare la propria condizione è un principio di conservazione capace di impedire e correggere sotto molti aspetti gli effetti negativi di un sistema economico in certo grado parziale e oppressivo". (Smith, 2006, p. 832). 
era sembrata attestarsi - specie con la edizione definitiva degli scritti di Ricardo curata da Piero Sraffa - su una ricostruzione del paradigma ricardiano. In realtà la cesura qui accennata è stata indebitamente e paradossalmente oscurata da una troppo intensa luce riservata al tema del valore: una luce da un lato troppo localizzata sul tema e dall'altro deformante, quasi una specie di iperbolica trasfigurazione la quale ha avuto larghissimo spazio all'interno della visione tradizionale della scuola classica. Come è noto la ricostruzione corrente della Scuola classica è, ancora oggi, molto condizionata da due elementi: da una parte vi è una presunzione di omogeneità tra Fisiocrazia e Scuola classica (come già accennato); dall'altra parte si è a lungo dato per acquisito il 'dogma' di una supposta stretta continuità tra Smith, Ricardo e Marx come traccia del percorso interno alla Scuola classica stessa.

Tutto questo va inteso alla luce di una realtà un poco più complessa.

Una linea di questo genere nel ricostruire la Scuola classica - che in realtà muove addirittura da William Petty, ossia dal Seicento inglese affonda le sue radici nella lettura offerta da Marx, soprattutto attraverso quella sua opera postuma (data alle stampe soltanto dopo l'inizio del secolo ventesimo) che è costituita dalla Teorie del plusvalore. Rimasta a lungo dormiente per effetto del declino della economia marxista tra la metà dell'Ottocento e la metà del Novecento, essa è stata ripresa e riproposta soprattutto da Piero Sraffa, come reazione rispetto alla linea interpretativa alternativa, di ispirazione neoclassica intesa a porre in luce nella Scuola classica gli elementi generatori del liberal-capitalismo, con una ricostruzione che include la esaltazione della mano invisibile, insieme a un concetto statico anziché dinamico di concorrenza.

La storia del pensiero economico, infatti, si è a lungo divisa tra due correnti circa l'influenza del pensiero classico: da un lato, un indirizzo di studi che vede in Ricardo il punto di maturazione massima del paradigma o canone classico secondo una concezione in realtà fatta propria e completata da Marx; dall'altro lato, vi è una concezione alternativa, emersa con la scuola neoclassica ossia con il marginalismo, che fa della Scuola classica l'incubatore della concezione neoclassica dell'equilibrio economico e del mercato. I punti di riferimento migliori in proposito possono essere ritrovati, per il primo paradigma nella opera di Piero Sraffa e per il secondo in Edwin Cannan. Nella concezione di Sraffa il primo paradigma rappresenta una sorta di identità perduta tutta da riscoprire, recuperare e sviluppare attraverso una strada rispet- 
to alla quale egli offre per primo l'esempio soprattutto con il suo schema lineare di produzione (uscito oltre cinquanta anni fa, nel 1960); per Cannan invece la Scuola classica rappresenta la origine imperfetta e remota della economia neoclassica e marginalista, già ben sviluppata e promettente al tempo nel quale egli scrive.

Occorre subito avvertire che entrambe queste presunzioni, riprese e sviluppate assai ampiamente nel corso del Novecento, sono sostanzialmente oggi superate dalla cosiddetta revisione storiografica operata soprattutto nei confronti di Smith. Non sono oggi più percorribili come tali e richiedono adattamenti e aggiornamenti molto sostanziali. Detta revisione ha infatti portato a riscrivere largamente la storia del pensiero economico classico.

Ciò premesso, il punto forse più importante in questa sede è costituito dal fatto che la direzione e il taglio adottato dalla revisione storiografica qui accennata non ha in realtà consentito di includere e tener conto a pieno titolo della tradizione italiana. Occorre dunque oggi aprire un nuovo fronte di indagine che si colloca alla intersezione tra il piano della storia delle idee e il piano della storia della analisi economica e che affronta il problema degli illuminismi, ossia delle diverse tradizioni di filosofia politica che occupano la scena specie nella seconda metà del Settecento, al fine di esaminare il loro rapporto con l'economia politica. E' in questo quadro che riteniamo di poter sostenere e illustrare come il risultato intellettuale più notevole dell'illuminismo italiano sia costituito dalla economia civile di Genovesi e seguaci. Sosteniamo inoltre che, proprio alla luce di quello sviluppo teorico, diventa oggi possibile mettere pienamente a fuoco e comprendere la necessaria cesura che divide autori come Smith e Ricardo.

Da un'altra prospettiva è ancora precisamente il pensiero italiano della seconda metà del Settecento che forma la radice e l'origine di un altro tipo di sviluppo, ancora certo connesso al punto precedente: ossia la costante 'distanza' della tradizione italiana rispetto alla nuova teoria del valore di Smith e di Ricardo, ossia dalla teoria del valore lavoro, che Marx trasformerà in teoria dello sfruttamento. Tale 'distanza', non di rado (soprattutto per quanto concerne il primo Ottocento) interpretata come un segno di arretratezza della tradizione italiana (per esempio anche da parte di Francesco Ferrara, la cui critica della tradizione italiana riguarda anche altri aspetti), è in realtà spiegabilissima se si segue con coerenza la linea di storia intellettuale tipica della stessa economia civile: proprio di essa tale 'distanza' è frutto tuttora insufficientemente 
riconosciuto. Questa prospettiva critica di fronte alla teoria del valore in generale, e più particolarmente rispetto all'opera di Marx, rappresenta una costante importante nel pensiero italiano, tanto da ritrovarsi perfino nelle elaborazioni italiane del marxismo di autori quali Loria, i Labriola, Croce fino a Graziadei e soprattutto a Sraffa. ${ }^{3}$

E' possibile mostrare che nella stessa direzione si colloca anche il risultato che emerge dalla concezione di economia dinamica alla quale, come si è accennato sopra, conduce la tradizione italiana e nella quale trova posto la stessa concezione della dinamica strutturale, frutto in realtà della simbiosi tra il pensiero italiano e la Scuola di Cambridge.

Il problema fondamentale di tutta l'economia moderna è còlto perfettamente dal titolo della opera più nota di Adam Smith, là dove si parla di indagine sulla natura e le cause della ricchezza delle nazioni. Una caratteristica fondamentale del contributo di Smith consiste nel collegare e coniugare quella indagine con la ricerca dei fondamenti dell'ordine sociale, ossia di una società bene ordinata, visto come condizione indispensabile alla crescita ossia all'arricchimento. Analisi di natura e cause sono in rapporto sinergico. Nell'introdurre il quarto libro, dedicato alla storia delle teorie economiche, Smith precisa il significato generale della analisi economica. "L'economia politica - scrive Smith - considerata come un ramo della scienza dello statista o del legislatore, si propone due oggetti distinti: primo, provvedere un abbondante reddito o sussistenza; secondo fornire lo stato o la repubblica di un'entrata sufficiente per i servizi pubblici. Essa si propone di arricchire sia il popolo che il sovrano". Egli poi aggiunge: "Il diverso progresso dell'opulenza in diverse epoche e nazioni ha dato origine a due diversi sistemi di economia politica relativamente al problema di arricchire la gente. Uno si può chiamare sistema commerciale, l'altro sistema agricolo". In realtà Smith sviluppa una critica radicale dei due sistemi e oppone a ciascuno di essi il proprio sistema, nel quale è presente nella sostanza la prospettiva del civile, a collocare la radice del progresso, ossia dell'arricchimento, nello sviluppo delle relazioni tra i soggetti.

Il passaggio dal mercantilismo al periodo 'classico' (inclusa la Fisiocrazia) può essere qui descritto nei suoi termini più generali come

3 Per quanto concerne Marx e il marxismo rinvio a Bellanca, 1997. Per la teoria del valore in generale, in rapporto alla tradizione italiana, Bruni e Porta, 2007, dove si afferma (p. 15) che in Italia già con Pareto "abbiamo la fine della stagione del valore nell'economia ufficiale". 
contrassegnato da una cesura molto netta che include i seguenti passaggi: 1. Rifiuto del concetto bullionista della pratica identificazione della ricchezza con l'accumulo di moneta e di strumenti finanziari; 2. Rifiuto del c.d. 'profit upon alienation', ossia della idea che l'eccedenza o prodotto netto sia frutto di guadagno conseguito attraverso lo scambio; 3. Generale adesione al principio della superiorità del laissez-faire, ossia carattere naturale del regime concorrenziale; 4. Rifiuto o superamento del popolazionismo; 5. Rifiuto della concezione monetaria dell'interesse, la quale presuppone a sua volta una nozione monetaria del capitale, che viene invece sostituita dalla nozione reale di capitale.

Questi passaggi avvengono nel pensiero italiano, secondo un itinerario parallelo a quello rintracciabile nella Scozia, in una forma meno drastica rispetto quanto accade in Francia. Sarebbe utile qui un richiamo (che per brevità omettiamo) alla letteratura del dopoguerra sull'Illuminismo, sino alle riletture recenti, da Franco Venturi a Jonathan Israel. Le prospettive di studio sull'Illuminismo, specie in relazione con il pensiero economico, possono infatti essere collocate entro il quadro della distinzione tra quello che chiamiamo qui illuminismo civile e l'illuminismo radicale sul quale si è diffusa molta letteratura recente. Di fatto questa distinzione ha prodotto il risultato di attirare l'attenzione soprattutto sopra l'illuminismo radicale come il sistema di pensiero che ha forgiato la maggior parte dei valori sociali e culturali della nostra epoca. In realtà una simile prospettiva implica una enorme sottovalutazione dell'illuminismo civile e della sua influenza, probabilmente dovuta proprio a una insufficiente attenzione al caso italiano, il quale si presenta come una delle espressioni più forti e coerenti dell'illuminismo civile. Sotto questo aspetto (pur con le necessarie qualificazioni e distinzioni interne) si può dire che diventa essenziale studiare il caso italiano separatamente dal caso francese.

Fondamentale è qui la analisi della idea di progresso che contraddistingue l'illuminismo civile. Si tratta di un progresso di tipo costituzionale che mira allo sviluppo di un insieme stratificato di istituzioni che aprirà in tempi successivi la strada al liberalismo. ${ }^{4}$ Lidea di fondo è quella di libertà. Per l'illuminismo radicale invece la idea di fondo è quella della uguaglianza e la finalità politica è il democratismo radicale.

4 E' questa una tesi che ha significativa eco, per esempio, in Capra, 2002, p. 291 e p. 383 . 
La disciplina economica diventa a partire soprattutto dal Settecento un pilastro fondamentale dell'idea dell'Illuminismo civile. Anche qui, limitando il campo al pensiero economico, vi sono delle differenze tra contesti diversi: nel caso francese, la reazione al Colbertismo conduce a esaltare il mercato e il laissez faire come il perno dell'ordine sociale. L'unica politica possibile è quella di mettere i mercati concorrenziali in condizioni di funzionare perché l'ordine di mercato è sufficiente di per sé per correggere gli squilibri della società. Nel caso italiano la concezione ha altri caratteri: tra economia e istituzioni c'è una compenetrazione assai forte. Il caso lombardo, Verri in particolare, è sicuramente interessante.

Occorrerà infine esaminare la continuità nel tempo della linea italiana, e lombarda in particolare, a cominciare con il primo Ottocento milanese. Si è spesso sostenuta la tesi di sterilità del pensiero economico italiano specie nel primo Ottocento, quasi che quella che qui è chiamata la 'linea italiana' (civile, o - come talvolta si afferma - moderata) sia stata schiacciata nello scontro perenne (e del resto tuttora in corso) tra illuminismo radicale e anti-illuminismo. In realtà il caso italiano, nel corso degli ultimi due secoli, può risultare istruttivo proprio per aver dato luogo a interessanti correnti di pensiero economico. Questa considerazione, per cominciare, è alla base della concezioni unitaria del periodo 1750-1850 e degli elementi di continuità della tradizione italiana fino al presente.

Esaminiamo ora i caratteri dell'Illuminismo lombardo: quelli almeno che sono alla origine della concezione dell'economico che fiorisce in Lombardia nella seconda metà del Settecento.

Antonio Genovesi è in Italia il maestro riconosciuto da tutti oltre che primo cattedratico della disciplina per l'influenza e i buoni uffici di Bartolomeo Intieri.

Punto centrale della economia civile inaugurata da Genovesi è la tensione tra due forze di segno opposto: forza concentriva e forza diffusiva (Stapelbroeck, 2007; Bruni e Sugden, 2007, in particolare pp. 139140). E' stato notato che, pur all'interno di una visione ispirata a forme di utilitarismo settecentesco, Genovesi è profondamente influenzato dalle impostazioni neo-platoniche (Shaftesbury) e dalle filosofie del senso morale (da Gershom Carmichael a Francis Hutcheson, il cui insegnamento verrà raccolto da Smith), con la caratteristica insistenza sulla pluralità dei sentimenti morali di segno non esclusivamente autointeressato. E' anche evidentemente influenzato dal linguaggio newtoniano. 
Una concezione dell'ordine sociale spontaneo che ha al suo centro l'idea della eterogenesi dei fini da un lato (e quindi derivante dalla influenza di Mandeville e dalla polemica nei confronti di Hobbes) e dall'altro l'idea antropologica di animal civile (ispirata a Vico e in continuità con gli umanisti civili ai quali si è fatto cenno) sono i fatti che rendono per la prima volta esplicita quella concezione cooperativa del mercato concorrenziale che troverà in Smith l'espressione più nota (si veda in proposito anche il volume di Robertson, 2005). Il taglio anticartesiano del verum ipsum factum (che radica la vera conoscenza nel progettare e nel fare) di Vico costituisce un importante elemento per comprendere la radice della economia civile. E' una linea argomentativa di questo genere che induce Genovesi a rovesciare il celebre adagio di Hobbes (bomo bomini lupus) nel suo contrario bomo bomini natura amicus (v. IIISF, 2007, p. 42). A volte gli si contrappone la frase bomo bomini deus: il significato non è dissimile (v. Natoli, Parole della filosofia, cap. 11). Di qui l'importanza che Genovesi attribuisce alla pietà, e che lo conduce a concepire la società umana ("civile") come fondata sul diritto dei suoi membri ad essere soccorsi. La concezione del mercato come forma di mutua assistenza darà notoriamente i suoi frutti nella Ricchezza delle nazioni.

L'insegnamento di Genovesi inaugura una stagione feconda del pensiero economico italiano che costituisce una rielaborazione di questa linea di indagine. Qui si inserisce il contributo della Scuola di Milano, quale trova espressione nella Accademia dei Pugni, nel Caffè e nelle opere degli autori collegati. Nella sede lombarda il tema della economia civile appare più esplicitamente calato in un confronto diretto con la realtà economica e con le esigenze pratiche di una politica delle riforme. E' quest'ultimo elemento che motiva, nel contesto milanese, la adozione di un taglio e un linguaggio in parte diverso rispetto al caso della Scuola napoletana.

\section{Pietro Verri: ECONOMIA E ISTITUZIONI}

In Pietro Verri, in particolare, oltre al rappresentante forse più eminente dell'eudemonismo settecentesco, dobbiamo vedere lo spirito pratico, con tratti squisitamente lombardi, del riformatore. Questo aspetto del pensiero e dell'azione di Verri si colloca in diretto rapporto con il ruolo della monarchia austriaca, dopo Aquisgrana, con il regno 
di Maria Teresa e, successivamente, con il regno dei figli di lei Giuseppe e Leopoldo.

Un'osservazione che spesso si fa in proposito è la seguente. E' assai facile, sulla base del dispiegarsi del moto riformatore all'interno della monarchia austriaca, esagerare l'importanza e l'influenza degli intellettuali lombardi alla corte viennese. Nella seconda metà del Settecento si inaridirono gli apporti culturali che l'Austria traeva dagli ambienti milanesi, all'interno di una più complessa contrazione dell'influenza italiana sulla vita intellettuale della Monarchia asburgica, che fu maggiormente animata da uomini di formazione tedesca, austriaca, boema, olandese. ${ }^{5} \mathrm{Se}$ si eccettua il caso di Beccaria, la cui opera si era prontamente imposta alla attenzione di tutta la cultura occidentale, il prestigio intellettuale dei lombardi - Verri incluso, considerato in realtà soprattutto per le sue proposte tecniche - fu più contenuto. "D'altra parte - aggiungono significativamente gli stessi autori - furono gli stessi illuministi lombardi a mantenere un rapporto strumentale con la Monarchia asburgica. I loro referenti ideali erano altrove: a Parigi, a Londra, nelle libere province americane, nella Corsica di Pasquale Paoli, là dove l'esperienza politica o la critica ideologica mostravano più direttamente la prefigurazione di un nuovo ordine sociale non più fondato sul dispotismo dei prìncipi". Anche alla luce di queste e simili considerazioni sembra di potere osservare che l'impostazione intellettuale di Pietro Verri è complessivamente assai più coerente con il suo tardo costituzionalismo piuttosto che con i suoi precedenti pronunciamenti a sostegno dell'assolutismo illuminato. Se una delle due posizioni deve essere vista quale frutto di istanze pratiche anzichè come posizione di princìpi, questa è piuttosto la prima posizione che non la seconda. Il punto è di qualche significato anche perchè l'iconografia corrente dipinge spesso un processo contrario: un Verri in anni giovanili fervente convinto sostenitore del modello assolutistico ripiegherebbe, deluso e scettico, sul modello costituzionale e sulla difesa di quei corpi intermedi e livelli intermedi di governo ch'egli aveva così tenacemente combattuto. $^{6}$

Tentiamo dunque di meglio definire la concezione di Pietro Verri, nella quale il ruolo fondamentale della concorrenza e del mercato si collega strettamente con la concezione della società civile. Vediamo infatti

5 Carpanetto-Ricuperati, 1986, p. 297.

6 Cfr., ad es., Valeri, 1937. 
in Verri una scienza del legislatore alla quale contribuisce la cultura francese (Montesquieu) e anglosassone (Locke, Hume), dove il legislatore compare come figura pubblica che codifica i nessi costitutivi della società civile; il discorso di Verri non si presenta nè come mero economicismo, nè come teorizzazione dell'uomo politico o del potere politico. Qui intervengono i caratteri di una tipica concezione della civitas che ha le sue radici appunto anche in Montesquieu e nella tradizione anglosassone. La lotta di Verri contro l'arbitrio e il privilegio non ha dunque nulla in comune con la teorizzazione dell'assolutismo politico, un elemento che per Verri ha un valore strumentale. ${ }^{7}$

Nella sua discussione dei governi dispotici, Montesquieu scrive: "Dopo tutto quello che abbiamo detto, sembrerebbe che la natura umana dovrebbe ribellarsi senza posa contro il governo dispotico. Eppure, nonostante l'amore degli uomini per la libertà, nonostante il loro odio per la violenza, la maggior parte dei popoli vi sono sottomessi. Ciò è facile da comprendere. Per formare un governo moderato bisogna combinare i poteri, regolarli temperarli, farli agire; dare, per così dire, un contrappeso a uno per metterlo in grado di resistere a un altro; è un capolavoro di legislazione che il caso fa di rado e che di rado è concesso di fare alla prudenza. Al contrario un governo dispotico salta, per così dire, agli occhi; è lo stesso dovunque: poichè per stabilirlo bastano le passioni, chiunque è capace di farlo".${ }^{8}$ La salvaguardia dal dispotismo è legata allo sviluppo del governo limitato dal potere supremo della legge. Nella concezione di Montesquieu il potere supremo della legge non può stabilirsi e perdurare se non vi sono istituzioni indipendenti - $\mathrm{i}$ pouvoirs intermédiaires - cui la legge attribuisce identità e poteri. E' questa una concezione che esalta la centralità alla società civile intesa come rete di rapporti sociali all'interno della quale si colloca la stessa autorità politica ossia l'autorità di governo. La filosofia politica al riguardo ci insegna a riconoscere un parallelo tra il più celebre capitolo dell'Esprit des lois - il sesto del libro undicesimo - e un testo sulla divisione dei poteri precedente di mezzo secolo e quasi altrettanto cele-

7 Si vedano in proposito Porta e Scazzieri, 2002; Porta, 2007. Si confronti anche con l'introduzione del curatore Renzo De Felice a Verri, 1964, spec. pp. xvi e segg. L'influenza di Montesquieu, specie per quanto concerne l'economia politica di Pietro Verri, è ivi sottolineata a p. 128.

8 L'esprit des lois, 1748, libro V, cap. xiv. 
bre, il capitolo dodicesimo del secondo trattato sul governo di John Locke. Entrambi i testi parlano della costituzione britannica.

Non è casuale che John Locke e il barone di Montesquieu siano tra le massime fonti di Pietro Verri; è, anzi, questo un fatto che qualifica la concezione costituzionalista di Verri, la quale, come si è detto è anzitutto parte della sua formazione filosofica prima di essere la fonte di concrete proposte di riforma giuspubblicistica all'epoca della rivoluzione francese e dopo la scomparsa dell'imperatore Giuseppe II. Sotto il profilo filosofico, del resto, proprio la congiunzione tra eudemonismo e società civile è all'origine della propensione di Pietro Verri per l'economia politica.

La concezione della legge e della attività legislativa elaborata da Pietro Verri è al riguardo fondamentale. La libertà politica è definita significativamente da Pietro Verri precisamente nei termini di libertà civile: "col nome di libertà politica - egli scrive - io intendo l'opinione che ha ogni cittadino di possedere se medesimo e quello che è suo e di poterne a suo piacere disporre sin tanto ch'ei non trasgredisca le leggi promulgate con legittima autorità". E ancora: "Il più dolce, il più benefico impero è quello delle leggi; esse non conoscono parzialità, non hanno affetti; sode, immutabili, ordinano lo stesso ad ognuno".?

Che la impostazione fondata sopra l'impero della legge, ossia la concezione del sovrano come soggetto alle leggi, sia circostanza non certo estranea allo sviluppo concettuale dell'economia politica è universalmente noto almeno dacchè Adam Smith ha posto all'inizio del quarto libro della Ricchezza delle nazioni la celebre definizione della political economy quale branca della scienza dell'uomo di stato e del legislatore. Non è difficile constatare come Verri preceda Smith sullo stesso itinerario, il quale fa del legislatore la figura nella quale è riassunto il funzionamento della società civile entro la quale il sistema economico si colloca, precisamente al fine di saggiarne il potenziale di crescita. ${ }^{10}$

9 “Sulla interpretazione delle leggi", in Caffè; ed. 1993, pp. 700 e 703.

10 "Political œconomy, considered as a branch of the science of a statesman or legislator, proposes two distinct objects: first, to provide a plentiful revenue or subsistence for the people, or more properly to enable them to provide such a revenue or subsistence for themselves; and secondly, to supply the state or commonwealth with a revenue sufficient for the public services. It proposes to enrich both the people and the sovereign”. (Dalla Introduzione al libro IV della Ricchezza delle Nazioni). 
La libertà - come già in Montesquieu, XI, 4 - è definita come soggezione alle leggi. "La grand'arte del legislatore - premette Verri - è di sapere ben dirigere la cupidigia degli uomini. Allora si scuote l'utile industria dei cittadini; l'esempio, l'emulazione e l'uso fanno moltiplicare i cittadini utili, i quali cercano a gara di farsi più ricchi col somministrare alla patria merci migliori a minor prezzo". E prosegue: "La libertà e la concorrenza sono l'anima del commercio; cioè la libertà che nasce dalle leggi, non dalla licenza. Quindi ne segue che l'anima del commercio è la sicurezza della proprietà fondata su chiare leggi, non soggette all'arbitrio; ne segue pure che i monopolj, ossia i privilegi esclusivi sieno perfettamente opposti allo spirito del commercio". ${ }^{11}$

Non è dunque la presenza di istituzioni o corpi intermedi l'obiettivo della critica di Pietro Verri rivolta a forme di privilegio corporativo. Il problema che egli pone è invece quello di impedire la formazione del privilegio e di liberarne eventualmente il sistema nel quale il privilegio abbia messo radici. Diversamente infatti si impedirebbe il raggiungimento del fine cui debbon tendere le operazioni della pubblica economia col "rendere difficile l'industria de' cittadini". Infatti i "corpi delle arti e dei mestieri non producono il bene per cui furono istituiti"; essi "tendono a diminuire l'annua riproduzione". Quest'ultimo è l'argomento ultimamente decisivo sul quale l'Autore poggia il richiamo contro "lo spirito di lega e monopolio" che sortisce l'effetto di "diminuire il numero dei venditori interni" con conseguente accrescimento del prezzo delle merci, diminuzione del numero dei contratti, freno all'industria e all'annua riproduzione. ${ }^{12} \mathrm{Il}$ rimedio sta allora nell'aprire "la strada ampia e libera a chiunque di esercitar la sua industria dove più vuole"; solo per tal via si vedrà "l'abbondanza scorrere dovunque guidata dalla concorrenza, inseparabile compagna di lei;... così nelle società accader deve che tutto prenda lena e vigore e si riscaldi, quando il desiderio di migliorare la sorte non incontri ostacolo, e possa per ogni dove spignersi e largamente e sicuramente signoreggiare" (ibid.). Il rimedio sta nella promozione di una buona e saggia legislazione; quando il privilegio è radicato può tuttavia ben essere necessario uno

11 "Elementi del commercio", in Caffè, ed. 1993, pp. 35-36. Il ms. orginario risale al 1760; pubblicato con poche variazioni nel Caffè, 1764; ristampato dal Custodi, 1804.

12 Verri, Meditazioni sulla economia politica, 1772, spec. $\$ 7$; Edizione Nazionale, Vol. II. 2, p. 450. 
speciale temporaneo intervento dell'autorità politica allo scopo di ristabilire la funzionalità del sistema.

Lungi dal forzare e prescrivere, le leggi debbono invitare e guidare; il principio regolatore è che "una legge, che abbia contro di sè la natura e l'interesse di molti, non può mai essere costantemente e placidamente osservata, nè portare fauste conseguenze alla città". " ${ }^{3}$ "La grand'arte del legislatore è, e sarà sempre quella di far coincidere l'interesse privato col pubblico". ${ }^{14}$ Gran parte dell'arbitrio, come dimostra la storia del milanese, risiede nell'accumulo di poteri e privilegi da parte non solo dei corpi intermedi, ma più precisamente dei livelli intermedi di governo. In particolare il codice promulgato sotto la dominazione spagnola è ispirato a princìi contrari alla libertà e all'industria; ivi si considerano "i senatori e i curiali come padroni della nazione, e non fatti per lei. Contro il principio stabilito da Montesquieu, in una stessa magistratura si è riunito il potere legislativo e il potere esecutivo. "In somma fu tolta dalle radici quella sicurezza civica che era una delle cagioni dell'antica prosperità; tutto divenne contenzioso, precario e incerto; l'arbitrio del giudice prevalse ad ogni legge; non fu più la provincia governata dalle leggi, e cominciò il governo degli uomini". ${ }^{\text {s }}$

Resta il fatto che condizioni particolari debbono riguardare quel mutamento di abitudini e di costumi che è il fine di una "felice rivoluzione". Ovunque si tratti della "esecuzione di leggi già fatte, è utile, anzi indispensabile il farne dipendere la decisione dalla opinione di più uomini; per lo contrario dove si tratta di organizzare sistemi e dirigere il corso a un determinato fine, sorpassando le difficoltà che si frappongono e che tutte non possono mai prevedersi, necessità vuole che quest'impeto e questa direzione dipenda da un solo principio motore". Si tratta di un arbitro di nuove regole operante in situazioni eccezionali, "siccome la dittatura fu appunto presso i Romani nelle cose ardue adoperata felicemente". In economia politica dunque, "singolarmente quando si tratti di ridurla a semplicità riformando i vecchi abusi”, vi è la necessità di "creare un dispotismo che duri quanto basta ad aver messo in moto regolarmente un provvido sistema" (Meditazioni, $\mathbb{\$} 38$ ).

13 Verri, ib., 1772, spec. $\ 12$; Edizione Nazionale, Vol. II. 2, p. 428.

14 Verri, Leggi vincolanti, 1796, \$5: v. Collezione Custodi, parte moderna, Vol. XVI, p. 131.

15 Verri, Memorie storiche, 1804, $\ 3$ : v. Collezione Custodi, parte moderna, Vol. XVII, p. 78. 
Costantemente reiterata è in Verri l'opinione che nelle "nazioni illuminate gli uomini vanno direttamente, e obliquamente vanno le leggi; ma quanto sono minori i lumi di un popolo, tanto vanno più direttamente le leggi e obbliquamente gli uomini".${ }^{16}$ Così nel campo dell'annona egli censura l'aspirazione dell'uomo volgare a ricondurre il vivere civile al limitato orizzonte della propria mente: "L'uomo volgare sempre più voglioso d'imprimere il moto alla società, e di crearvi qualche cosa del suo, anzi che indirettamente incanalare e rendere cospiranti le azioni, colle sue mani grossolanamente tasteggia e scompone la gran macchina della civile società, gli ordigni sottilissimi della quale gli sfuggono dallo sguardo; ma l'uomo che ha meditato vede che nessuna nazione è tanto stolida da privare se stessa del necessario alimento, a meno che per un artificioso sistema di vincoli non succeda un rigurgito contrario alla natura; vede che nella politica più giova il lasciar fare che il fare" ${ }^{17}$ Da queste considerazioni Pietro Verri trae anche, in chiusura delle Meditazioni del 1771, il "carattere di un ministro di economia". Suo primario compito è quello di "[r]imuovere gli ostacoli, abolire i vincoli, spianar le strade alla concorrenza animatrice della riproduzione, accrescere la libertà civile, lasciare un campo spazioso all'industria, proteggere la classe dei riproduttori con buone leggi...; assicurare un corso facile, pronto i disinteressato alla ragione de' contratti; dilatare la buona fede del commercio col non lasciare mai impunita la frode". La formula "attivo nel distruggere, cautissimo nell'edificare" riassume, correttamente intesa, quello che potremmo chiamare il moderatismo politico di Pietro Verri e, insieme, la sua concezione dell'ordine sociale.

C'è una sorta di continuità tra Verri e Genovesi nella enfasi sulla importanza di arricchire in termini di maggiore prossimità e più intensa interazione. "Come si animi l'industria avvicinando l'uomo all'uomo" egli titola al $\$ 26$. Nello stesso senso opera la sua critica al sistema fisiocratico, là dove Verri argomenta che l'accrescimento del reddito e della ricchezza $(\$ 2)$ dipende dalla capacità di accostare l'uomo all'uomo. Di qui l'importanza del commercio e la critica dunque a quella scuola che ha "creduto che l'accrescimento del commercio fosse nocivo ai progressi dell'agricoltura", venendo così a negare gli effetti positi-

16 Verri, Meditazioni sulla economia politica, 1772, spec. $\$ 27$; Edizione Nazionale, Vol. II. 2, p. 511.

17 Verri, 1796, parte prima; ed. Custodi, p. 40. 
vi dell' "instancabile industria" e del "florido commercio" $(\$ 13)$ nel raffinare le arti, espandere i bisogni e stimolare la creatività. "La classe adunque de' manofattori non può dirsi sterile" (\$ 3 ). Si è notato (v. IISF, p. 132, saggio di Bruni e Sugden) come Gaetano Filangieri, riprendendo questa analisi di Verri, abbia parlato del commercio come espressione dello spirito di fraternità.

Tutto questo spiega la posizione che sarà di li a breve espressa da Smith nella quale divisione del lavoro e commercio sono due facce di una medesima realtà, ossia della realtà di una società che arricchisce con il rendere sempre meno autosufficienti i suoi membri. Una volta affermatasi la divisione del lavoro ciascuno col proprio lavoro si procura una parte minima dei mezzi per vivere. L'uomo - scriverà Smith - ha costantemente bisogno dell'aiuto dei suoi simili né può pensare di procurarselo facendo semplice appello alla loro benevolenza.

\section{L'ECONOMIA CIVILE NEL PARADIGMA CLASSICO IN ECONOMIA}

Vi è una stretta continuità tra la economia civile elaborata dagli illuministi in Italia e la economia politica di Adam Smith. Entrambe le forme della teoria economica attingono al dibattito europeo: Hobbes, la teoria del 'senso morale', Mandeville, Montesquieu, Hume.

In Italia il quadro concettuale dominante è quello dell'utilitarismo. Nel caso milanese il linguaggio economico-giuridico (Verri, Beccaria) è caratteristico dell'impegno attivo per la riforma della società e dello stato, mentre a Napoli prevale il linguaggio economico-filosofico. Pel caso milanese la presenza di Beccaria è di significativa continuità rispetto alle concezioni di taglio 'scienza del legislatore' che possiamo rintracciare (come abbiamo qui ricordato) in Verri.

Dopo Smith, invece, l'economia politica classica cambierà pelle con il passaggio a una impostazione pessimistica del tema valore-distribuzione, che avrà la sua maggiore espressione in David Ricardo.

Nelle riprese odierne del pensiero economico il percorso si è compiuto a ritroso: da un ritorno ai classici ispirato al ricardismo si sta passando a una concezione che mette al centro il paradigma smithiano.

Questo percorso riflette una linea di indagine caratteristica della tradizione italiana in economia politica, la quale dà la maggiore continuità alla idea guida della economia civile, mentre si tiene lontana dalla 'rigidità' del pensiero economico francese (gli 'economisti'). 
E' dunque essenziale osservare che la revisione storiografica che ha per oggetto il pensiero di Smith deve essere completata attraverso la riscoperta di una fonte importante del pensiero di Smith e dell'economia dell'illuminismo scozzese in generale. Ma non è questo soltanto l'elemento qui rilevante. E' infatti da rilevare come l'illuminismo civile italiano costituisca anche la radice della linea di indagine prevalente perseguita dal pensiero economico italiano nel corso dell'Ottocento e del Novecento.

\section{L'ECONOMIA CIVILE COME PRODOTTO DEL CANONE ILLUMINISTICO: LA CONTRAPPOSIZIONE ALL'ILLUMINISMO RADICALE}

Jonathan Israel ha sviluppato ampia analisi del concetto di Illuminismo radicale. E' un concetto che parte filosoficamente soprattutto dall'unitarismo spinoziano, anche se coinvolge in primo piano anche diversi altri autori, e si dipana attraverso una lunga serie di saggisti e teorici della politica e della morale tra i quali spiccano Holbach, La Mettrie, Helvétius. Secondo Israel l'Illuminismo radicale, anticlericale e anti-deista, deve essere studiato come il fenomeno più importante che si colloca alle radici della democrazia moderna. Per quanto concerne la fioritura di studi economici nell'epoca illuministica, secondo Israel, l'economia classica - che egli identifica essenzialmente con Turgot, Beccaria e Adam Smith - "per essere compresa in modo appropriato nel suo contesto storico, deve essere osservata contro lo sfondo della lotta tra il pensiero dell'Illuminismo radicale e quello dell'Illuminismo moderato" (p. 100).

"L'Illuminismo radicale - spiega Israel (p. vii) - è costituito da un insieme di principi di base che possono sinteticamente essere riassunti nei termini: democrazia, eguaglianza razziale e sessuale; libertà individuale nello stile di vita; piena libertà di pensiero, espressione e di stampa; sradicamento dell'autorità religiosa dal processo legislativo e dall'istruzione e piena separazione tra chiesa e stato". L'importanza che l'autore attribuisce al paradigma non è sottaciuta: "L'Illuminismo radicale è il sistema di pensiero che, storicamente, ha forgiato in modo prevalente la maggior parte dei valori sociali e culturali basilari nell'era postcristiana" (p. x). Nell'esprimere la sua valutazione critica circa l'idea di 'famiglia di illuminismi' sviluppata da John Pocock, l'a. sostiene che, 
"oltre un certo livello c'erano e ci potevano essere soltanto due tipi di Illuminismo: quello moderato (due sostanze), da una parte, che postulava l'equilibrio tra ragione e tradizione, e che appoggiava largamente lo status quo e, dall'altra parte, l'Illuminismo radicale (una sostanza), che univa corpo e anima in una cosa sola, riducendo Dio e la natura alla stessa cosa, escludendo tutti i miracoli e gli spiriti separati dal corpo, che invocava la ragione come unica guida della vita dell'uomo, abbandonando la tradizione" (p. 18).

Qui si inserisce l'economia classica, il cui elemento caratteristico è costituito dalla libertà di scambio. Il progresso sociale, in altre parole, è garantito dal laissez-faire. "La società progredirà e migliorerà se le leggi del mercato potranno operare liberamente e senza ostacoli. Tale miglioramento è raggiungibile ... senza interferire con i princìpi del rango e delle aristocrazia, o sfidare la monarchia e le autorità religiose; il mercato è sufficiente di per sé per correggere gli squilibri e i problemi della società" (p. 100).

La linea moderata dell'Illuminismo non merita, secondo Israel, gli onori che si è attribuita (p. 112): "i riformatori moderati, gli économistes e lo stesso Turgot presto avrebbero fallito, e fallito in modo spettacolare" (p. 112). La linea moderata sarebbe risultata, storicamente, schiacciata nello scontro tra i philosophes e gli anti-philosophes, tra l'illuminismo radicale e l'anti-illuminismo.

Vi però in questa argomentazione una completa sottovalutazione della differenza tra i diversi illuminismi riassobiti in questo caso entro il canone francese degli économistes. La differenza che Israel 'salta', senza neppure avvertirla, è la differenza tra l'imperialismo economico degli économistes e la concezione italo-scozzese della economia civile.

In questo modo il pensiero economico viene respinto in una posizione di secondo piano.

\section{TEORIA DINAMICA ED ECONOMIA CIVILE NEL PENSIERO ECONOMICO ITALIANO}

Abbiamo osservato come pensiero economico classico ed economia civile tornino a essere oggi categorie di avanguardia per l'analisi economica.

Nel suo più recente volume, Luigi Pasinetti raccomanda di volgere lo sguardo al passato, mirando al futuro. 
Appare dunque - e forse neppure troppo sorprendentemente che nella parte oggi più viva e costruttiva della maggiore Scuola di economia teorica, la Scuola di Cambridge, riaffiorano contenuti ispirati alla tradizione italiana. In questa sezione conclusiva l'obiettivo è quello di illustrare il senso - o quanto meno di discutere una possibile interpretazione - di quanto Luigi Pasinetti ha recentemente scritto in apertura della edizione italiana del suo recente volume qui già citato.$^{18} \mathrm{E}$ ' un fatto che "la 'rivoluzione keynesiana' - scrive Pasinetti - non riuscì a far cambiare il modo di pensare della maggioranza degli economisti teorici". Certo di "fondamenti teorici si era occupato ... Piero Sraffa, mente critica superba .... Ma non è bastato un approccio iper-critico, anche se penetrante" (op. cit., pp. xii-xiii; c.vo agg.). Qui sta la motivazione di fondo del volume di Pasinetti, il quale "è una continuazione della severa critica alla teoria dominante, ma vuol essere anche e soprattutto lo slancio costruttivo del proporre una teoria alternativa" (ib., p. xiii). Diventa chiaro il senso del volgere lo sguardo al passato, mirando al futuro, come Pasinetti scrive (ib., p. xv): ma nasce anche l'esigenza di approfondire il discorso e ricercare più precisamente quelle fonti che consentono a Pasinetti di rilanciare il tema della re-interpretazione della 'Scuola di Cambridge' con prospettive e significati in buona parte radicati in caratteri e direzioni specifiche dell'itinerario concettuale di questo autore.

Oggi si torna a parlare di 'Economia civile'. Se l'immagine consolidata della 'Scuola di Cambridge' appare superficialmente lontana dalla prospettiva della economia civile, ciò si deve al criterio riduttivo col quale essa è stata vista, specie nella sua relazione con l'Italia. In realtà le origini di quella necessaria 'congiunzione' sono da ritrovare nell'epoca classica e soprattutto nel legame che unisce l'Illuminismo italiano con l'Illuminismo scozzese nella seconda metà del Settecento. Il richiamo alla tradizione classica non stupisce certo nella ricostruzione della 'Scuola di Cambridge', e acquista anzi un senso preciso proprio alla luce della linea di pensiero elaborata da Pasinetti nel corso degli anni. ${ }^{19}$

18 Pasinetti, 2010, pp. xi-xiv.

19 Non a caso quello che può forse essere considerato il volume più importante di Pasinetti (1981) reca nel sottotitolo una chiara eco smithiana, che in parte differenzia la linea di analisi di questo autore rispetto ad altri filoni della 'Scuola di Cambridge'. 
Come già abbiamo ampiamente ricordato, l'idea della economia civile emerge nello sviluppo della disciplina economica dapprima con il corso universitario (il primo al mondo) delle Lezioni di commercio o sia di economia civile di Antonio Genovesi (1765-67). L'economia politica, nella modernità, aveva proceduto attraverso il passaggio da una logica di acquisizione fondata sul commercio, la banca e la finanza (Mercantilismo), ad una di produttività fondata su produzione e circolazione (Fisiocrazia), ad una logica infine di creatività fondata sull'apprendimento e il capitale umano (con le Scuole italiane dapprima e poi con Adam Smith). E' in questa ultima fase che acquista nuovo valore il tema della fiducia, della relazionalità e del rapporto dell'economia col mondo delle istituzioni. L'economia civile caratterizza in modo essenziale l'Illuminismo italiano.

L'ambiente intellettuale italiano, specie napoletano, è pervaso dall'interesse per il rapporto sociale (oggi diremmo la socialità o la relazionalità), la 'fede pubblica' capace di generare ordine sociale. L'esperienza milanese sviluppa temi similari, prendendo le mosse da una istanza pratica di conoscenza empirica che mira a predisporre gli elementi per una politica di riforma. E' da ricordare l'esperienza della generazione dei Verri e dei Beccaria. Nell'esperienza milanese alla istanza pratica si unisce una vasta rielaborazione concettuale che elabora una concezione della felicità pubblica.

A questi precedenti e a questo contesto occorre rifarsi per capire oggi la Rivoluzione che attende l'economia politica oggi. E' a questo riguardo che va fatto il collegamento con la 'Scuola di Cambridge'. L'esperienza intellettuale di Pasinetti, in particolare, rende evidente il limite di una logica del sovrappiù slegata dalle sue implicazioni di dinamica economica e impreparata a fornire punti di incontro per lo studio delle istituzioni della società civile. ${ }^{20}$

Specie a proposito di studio delle istituzioni, il fondamento della analisi di Luigi Pasinetti risiede in quello che egli chiama il teorema di separazione, a separation theorem, in virtù del quale (egli scrive) si afferma che "una separazione è necessaria tra le indagini che riguardano le

20 Un aspetto connesso riguarda la componente storico-analitica come elemento essenziale e qualificante della impostazione di Luigi Pasinetti, che costituisce un ulteriore elemento della interazione tra la tradizione italiana e la 'Scuola di Cambridge'. Si veda in particolare Porta, 2005. 
basi fondative delle relazioni economiche - che devono essere individuate a uno stadio strettamente essenziale - e le indagini che devono essere svolte al livello delle effettive istituzioni" (Pasinetti, 2010, p. 245). Le indagini del primo tipo riguardano le relazioni economiche fondamentali definite $\mathrm{e}$ individuate indipendentemente da specifici modelli comportamentali e assetti istituzionali. Questo è il livello di indagine che Pasinetti chiama 'naturale' e che consente la determinazione delle grandezze economiche "fondamentale" (ib.).

In virtù del 'teorema di separazione', l'impostazione di Pasinetti riesce ad abbracciare tutta una serie di nuovi elementi e, tra gli altri, proprio quegli elementi che rientrano nell'ambito della economia civile di autori come Bruni e Zamagni o Gui e Sugden e che sono tanta parte della tradizione italiana dall'Umanesimo a oggi. ${ }^{21}$ Alla struttura di legami di necessaria compatibilità espressa dal concetto classico di 'sistema naturale' viene associato - a un livello di analisi separato - lo studio delle istituzioni (ossia delle 'regole del gioco') necessarie per affrontare questioni localmente e storicamente specifiche del funzionamento del sistema economico.

In conclusione, tra gli studi recenti sulla economia civile che appaiono significativi nella prospettiva del presente saggio, vorrei qui menzionare in chiusura alcuni contributi di Alberto Quadrio Curzio, specie nel recente volume (Quadrio Curzio, 2007), che è particolarmente utile per illustrare l'aspetto della continuità della prospettiva di 'economia civile' che percorre l'intera tradizione italiana di pensiero economico. ${ }^{22}$ Non può sorprendere che lo stesso Alberto Quadrio Curzio, nel passare a trattare della esperienza formativa degli economisti italiani in questo dopoguerra, riconosca significativi elementi della tradizione italiana proprio nella analisi e nell'opera di Luigi Pasinetti. ${ }^{23}$

21 Il tema è svolto anche in Porta e Scazzieri, 2008, specie pp. 475-477.

22 Si veda in particolare A. Quadrio Curzio, "Cesare Beccaria e Pietro Verri: l'economia civile per il governo della 'cosa pubblica'", in Economisti ed economia. Per un'Italia europea: paradigmi tra il XVIII e il XX secolo, Bologna, Mulino, 2007, pp. 43. 56. Inoltre "L'identità e l'eredità economico-civile di una Accademia plurisecolare" (con C. Rotondi e M. Talamona), ibidem, pp. 117-152. L'intero volume di Quadrio Curzio può essere letto come una efficace e ampia illustrazione della tradizione italiana nella prospettiva delineata anche nel presente saggio.

23 Si veda A. Quadrio Curzio and C. Rotondi, "Sulle ricerche di economia politica in Cattolica" in G. Garofalo e A. Graziani, eds., La formazione degli economisti in Italia, Bologna: Mulino, 2004, cit., pp. 361-422, specie alle pp. 406-7. 
In particolare, come importante ingrediente del significato da attribuire al termine-concetto di 'economia civile', insistiamo qui sul fatto che il natural economic system di Luigi Pasinetti esclude ogni pretesa o volontà di endogenizzare le istituzioni.

L'analisi di Quadrio Curzio riveste anche significato nel tracciare paralleli importanti con altri autori della tradizioni italiana di economia politica. 


\section{BIBLIOGRAFIA}

\section{FONTI PRIMARIE}

Beccaria, C., 1994, Dei delitti e delle pene, a cura di Franco Venturi, Torino, Einaudi (prima edizione 1764).

"Il Caffè". 1764-66, ristampa a cura di G. Francioni e S. Romagnoli, Torino, Boringhieri, 1993.

Edizione Nazionale delle Opere di Cesare Beccaria, (a cura di Luigi Firpo e Gianni Francioni), Milano, Mediobanca (in corso di pubblicazione dal 1984).

Edizione Nazionale delle Opere di Pietro Verri, diretta da Carlo Capra e pubblicata in Roma, Edizioni di Storia e Letteratura. Vol. III: I "Discorsi" e altri scritti degli anni settanta, a cura di Giorgio Panizza, 2004; vol. II: Scritti di economia, finanza e amministrazione, a cura di Giuseppe Bognetti, Angelo Moioli, Pier Luigi Porta, Giovanna Tonelli (in due tomi), 2007-08.

Galiani, Ferdinando, 1975, Opere, a cura di Furio Diaz e Luciano Guerci, MilanoNapoli, Ricciardi.

Genovesi, Antonio, 2005, Delle Lezioni di Commercio o sia di Economia Civile con Elementi del Commercio, a cura di M.L. Perna, Napoli, Istituto Italiano per gli Studi Filosofici.

Smith, A., 2006, La ricchezza delle nazioni, ed. ital. a cura di A. e T. Bagiotti, Torino: Utet Libreria.

\section{FONTI SECONDARIE}

Barucci, P., 2009, Sul pensiero economico italiano (1750-1900), a cura di R. Patalano, Napoli, Istituto Italiano per gli Studi Filosofici.

Bellanca, N., 1997, Economia politica e marxismo in Italia, Milano, Unicopli.

Bellanca, N., 2000, Dinamica economica e istituzioni, Milano, Angeli.

Bruni, L., Porta, P.L., 2003, "Economies in the Age of Newton. Economia civile and pubblica felicità in the Italian Enlightenment", in collab. with L. Bruni, in the Annual Supplement to Vol. XXXIV (2002) of History of Political Economy, Durham: Duke Un. Press, 2003, pp. 261-286.

Bruni, L., Porta, P.L., 2007, "La rivoluzione mancata. La teoria del valore nei manuali degli economisti italiani”, in Augello, M.M., Guidi, M.E.L., eds., L'economia divulgata. Stili e percorsi italiani (1840-1922), Vol. II ("Teorie e paradigmi”), Milano, Angeli, pp. 3-20.

Bruni, L., Sugden, R., 2007, "Quale socialità in economia? Genovesi e Smith, economia civile e economia politica a confronto", in B. Jossa, R. Patalano, E. Zagari, a cura di, Genovesi economista: nel $250^{\circ}$ anniversario della cattedra di commercio e meccanica, Napoli, Arte Tipografica, pp. 125-154.

Bruni, L., Zamagni, S., 2004, Economia civile. Efficienza, equità, felicità pubblica, Bologna, Mulino.

Capra, C., 2002, I progressi della ragione. Vita di Pietro Verri, Bologna, Il Mulino. 
Carpanetto, D., Ricuperati, G. (1986), L'Italia nel Settecento. Crisi, Trasformazioni, Lumi, Bari, Laterza.

Dizionario di Economia Civile, 2009, Bruni L., Zamagni, S., eds., Roma, Città Nuova.

Faucci, R., 2000, Storia del pensiero economico in Italia, Torino, UTET Libreria.

Garin, E., 1993, L'umanesimo italiano. Filosofia e vita civile nel Rinascimento, RomaBari, Laterza.

Israel, J., 2011, Una rivoluzione della mente. L'illuminismo radicale e le origini intellettuali della democrazia moderna, Torino, Einaudi.

Istituto Italiano Studi Filosofici, 2007, Genovesi economista, a cura di B. Jossa. R. Patalano, E. Zagari, Napoli.

Natoli, S., 2004, Parole della filosofia o dell'arte di meditare, Milano, Feltrinelli.

Negri Zamagni, V. e Porta P.L., 2012, a cura di, Il contributo italiano alla storia del pensiero. Economia, Roma, Istituto della Enciclopedia Italiana.

Pasinetti, L.L., 2010, Keynes e i Keynesiani di Cambridge, Roma-Bari, Laterza.

Porta, P.L., 2005 “The History of Economics. Highlights from the Italian Tradition", in Dinamica economica e istituzioni. Studi in onore di Davide Cantarelli, a cura di A. Agnati, G. Marangoni, A. Montesano, A. Pellanda, Padova, Cedam, pp. 387-399.

Porta, P.L., 2007, Introduzione a Edizione Nazionale delle Opere di Pietro Verri, Scritti di economia, finanza e amministrazione, vol. II.2, pp. 1-91.

Porta, P.L., Scazzieri, R., 2002, "Pietro Verri’s Political Economy: Commercial Society, Civil Society, and the Science of the Legislator", in History of Political Economy, XXXIV, 1, March 2002, pp. 81-108.

Porta, P.L., Scazzieri, R., 2008, "A Revolution to be Accomplished. Keynes and the Cambridge Keynesians", Economia politica. Journal of analytical and institutional economics, n. 3, dicembre, 2008, pp. 455-480.

Quadrio Curzio, A., ed., 1996, Alle origini del pensiero economico in Italia. Economia e istituzioni: il paradigma lombardo tra $i$ secoli XVIII e XIX, Bologna, Mulino.

Quadrio Curzio, A., 2007, Economisti ed economia. Per un'Italia europea: paradigmi tra il secolo XVIII e il XX secolo, Bologna, Mulino.

Reinert, S. A., 2011, Editing and Introduction to Antonio Serra's Short Treatise of 1613, London, Anthem Press, pp. 1-96.

Robertson, J., 2005, The Case for the Enlightenment. Scotland and Naples 1680-1760, Cambridge, Cambridge University Press.

Silvestri, P., 2008, Il liberalismo di Luigi Einaudi o del Buongoverno, Soveria Mannelli, Rubbettino.

Stapelbroek, K., 2007, "L'economia civile e la società commerciale: Intieri, Genovesi, Galiani e la paternità dell'illuminismo napoletano", in B. Jossa, R. Patalano, E. Zagari, a cura di, Genovesi economista: nel $250^{\circ}$ anniversario della cattedra di commercio e meccanica, Napoli, Arte Tipografica, pp. 39-50.

Todeschini, G., 2004, Ricchezza francescana. Dalla povertà volontaria alla società di mercato, Bologna, Mulino.

Valeri, N., 1937, Pietro Verri, Milano, Mondadori.

Venturi, F., 1969, "La Lombardia delle riforme", in Settecento riformatore, vol. V, L'Italia dei lumi (1764-1790), pp. 425-594, Torino, Einaudi. 
Venturi, F., 1978, "Le Meditazioni sulla economia politica di Pietro Verri. Edizioni, echi e discussioni”, Rivista storica italiana, XC, iii, pp. 530-94.

Venturi, F., 1983, "Scottish Echoes in $18^{\text {th }}$ Century Italy", in Hont, I., Ignatieff, M., a cura di, Wealth and Virtue, Cambridge, Cambridge University Press, 1983, pp. 345-62.

Verri, P., 1964, Del piacere e del dolore ed altri scritti di filosofia ed economia, a cura di R. De Felice, Milano, Feltrinelli.

Winch, D., 1978, Adam Smith's Politics. An Essay on Historiographic Revision, Cambridge, Cambridge University Press. 